\title{
Epithelial Stem Cells: Making, Shaping and Breaking the Niche
}

\author{
Paula Ferraces-Riegas, Anona C. Galbraith, and David P. Doupé
}

\section{Abstract}

Epithelial stem cells maintain tissues throughout adult life and are tightly regulated by their microenvironmental niche to balance cell production and loss. These stem cells have been studied extensively as signal-receiving cells, responding to cues from other cell types and mechanical stimuli that comprise the niche. However, studies from a wide range of systems have identified epithelial stem cells as major contributors to their own microenvironment either through producing niche cells, acting directly as niche cells or regulating niche cells. The importance of stem cell contributions to the niche is particularly clear in cancer, where tumour cells extensively remodel their microenvironment to promote their survival and proliferation.

\section{Keywords}

Cancer $\cdot$ Epithelia $\cdot$ Extracellular matrix . Homeostasis $\cdot$ Niche $\cdot$ Signalling $\cdot$ Stem cells

Authors Paula Ferraces-Riegas and Anona C. Galbraith have equally contributed to this chapter.

P. Ferraces-Riegas, A. C. Galbraith, and D. P. Doupé $(\bowtie)$ Department of Biosciences, Durham University,

Durham, UK

e-mail: david.p.doupe@durham.ac.uk

\section{Abbreviations}

BMP Bone morphogenetic protein

CAF Cancer-associated fibroblast

CSC Cancer stem cell

DMBA 7,12-Dimethylbenz(a)anthracene

EC

ECM Extracellular matric

EE Enteroendocrine

EGF Epidermal growth factor

EGFR Epidermal growth factor receptor

FGF Fibroblast growth factor

ISC Intestinal stem cell

JAK/STAT Janus kinase/signal transducer and activator of transcription

SOS Son of Sevenless

TA Transit amplifying

TIC Tumour-initiating cell

TPA 12-O-tetradecanoylphorbol-13acetate

VEGF Vascular endothelial growth factor

\section{Introduction}

Adult tissue stem cells play critical roles throughout the body in maintaining normal tissue homeostasis and responding to challenges such as damage. The stem cells must generate sufficient cells to maintain the tissue without overproliferating, which is a hallmark of cancer (Hanahan and Weinberg 2017). Stem cells are therefore 
tightly regulated by their local microenvironment or niche. As misregulation of stem cells has major implications for both normal physiology and diseases such as cancer, a great deal of research has focused on understanding the stem cell niche. Classically, the niche has been thought of in terms of signalling from adjacent cells, but more recent work has led to an expanded definition that incorporates not only classical signalling pathways but also mechanical and metabolic inputs (Scadden 2014; Chacón-Martínez et al. 2018).

Even as understanding of what constitutes the niche has broadened, most studies have focused on the cells and tissues surrounding the stem cells and their role in creating the microenvironment. In this context, stem cells are viewed primarily as the recipients of chemical and mechanical cues. However, work in a range of systems has shown that stem cells themselves contribute substantially to the niche. In this review, we will take a stem cell-centric view of the niche and explore the roles of stem cells themselves in producing and regulating their niches. Taking examples of epithelial stem cells from a range of model organisms and tissues, we will discuss the roles of stem cell progeny, autocrine stem cell signals and stem cell regulation of the niche in tissue homeostasis and repair. We will also consider how these principles of stem cell niche regulation are subverted by cancer cells to enable overproliferation, invasion and metastasis with implications for therapeutics and prognostic biomarkers.

\section{$2 \quad$ Making the Niche: Stem Cell Progeny as Niche Components}

While niche signals often originate from surrounding tissues, such as the underlying mesenchyme for many epithelial stem cells, an increasing number of examples have emerged of epithelial stem cell progeny playing a major role as sources of niche signals (Fig. 1a). In the mammalian intestine, for example, both mesenchymaland stem cell-derived epithelial cells supply signals to regulate the intestinal stem cells
(ISCs) (Santos et al. 2018). The epithelium is maintained by multipotent Lgr5+ stem cells located in the spatially restricted crypts of Lieberkuhn at the base of the villi (Barker et al. 2007). ISCs generate both absorptive enterocyte (EC) cells and secretory cells, including enteroendocrine (EE) cells and Paneth cells. In the crypt base, stem cells are found intercalated between large Paneth cells with which they form extensive surface contacts (Sato et al. 2011). Expression profiling showed Paneth cells express many ligands for pathways involved in stem cell regulation in vivo, including EGF, Dll4 and Wnt3, and depletion of Paneth cells can result in reduced stem cell numbers (Sato et al. 2011). Further evidence for the niche role of Paneth cells comes from the growth of self-organizing intestinal organoid cultures in vitro. These organoids develop with the support of appropriate growth factors in the absence of mesenchymal cells, but the efficiency of their growth is enhanced by the addition of Paneth cells (Sato et al. 2009, 2011).

Other studies have shown that stem cell function can be retained in the absence of Paneth cells, suggesting a degree of redundancy in the sources of niche signals (Durand et al. 2012; Kim et al. 2012). Non-epithelial cell types, including telocytes and discrete subtypes of mesenchymal cells, are also sources of ligands such as Wnts (Aoki et al. 2016; Valenta et al. 2016; Degirmenci et al. 2018; Shoshkes-Carmel et al. 2018). While the relative importance of these sources of niche signals may be unclear, Paneth cells, produced by the ISCs, make a significant contribution to the stem cell niche. Indeed, increased mTORC1 activity in Paneth cells with age leads to elevated expression of the Wnt inhibitor Notum, reducing stem cell proliferation, which demonstrates that changes in stem cell progeny can impact the niche and hence stem cell fate (Pentinmikko et al. 2019).

The simpler intestinal model of the Drosophila midgut is also maintained by a population of ISCs that generate both absorptive EC and secretory EE cells (Micchelli and Perrimon 2006; Ohlstein and Spradling 2006). While a direct equivalent of the Paneth cells is absent, both progeny types 
A

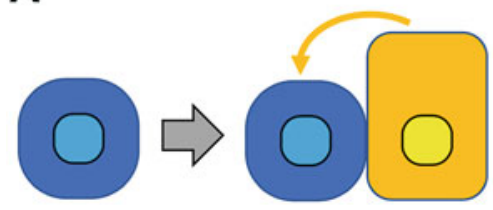

C

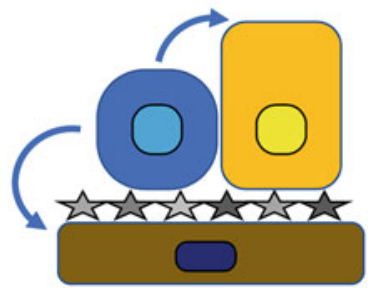

Fig. 1 Stem cell niche contributions from stem cells (a) Stem cells (blue) may generate progeny (yellow) that act as niche components regulating their own progenitor stem cells

(b) Stem cells may act as their own niche cells producing autocrine signals or ECM components (grey stars)

(c) Stem cells may regulate epithelial or non-epithelial
B

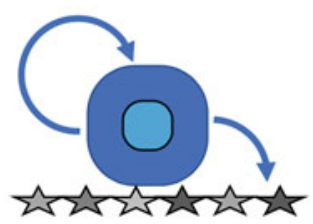

D

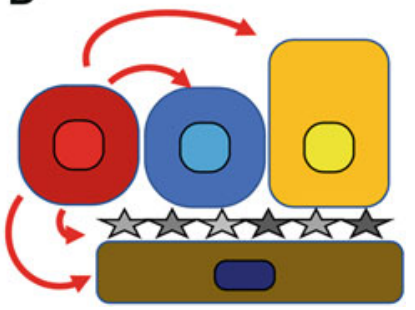

(brown) niche cells or the ECM to influence their own environment

(d) Cancer cells (red) may subvert the niche, exploiting any and all of the approaches $\mathbf{a}-\mathbf{c}$ to create a microenvironment that favours their growth at the expense of normal cells

expressed by differentiating EBs function redundantly to regulate the ISCs (Biteau and Jasper 2011; Jiang et al. 2011; Xu et al. 2011). Regulated secretion of EGF ligands from ECs is instrumental in driving turnover (Liang et al. 2017). These ligands are expressed in membrane-bound precursor forms that are unable to signal, but in response to EC apoptosis, the protease rhomboid is induced and cleaves the precursors to allow secretion of active EGF ligands. The resulting EGFR signalling in ISCs drives their proliferation (Liang et al. 2017), demonstrating the utility of feedback from progeny to the stem cells in coupling cell loss and replacement to maintain balance.

A similar coupling of differentiated progeny cell loss to stem cell proliferation has been demonstrated in the mouse epidermis. Live imaging studies were used to track thousands of nuclei in the basal layer of the epidermis over time (Mesa et al. 2018). It was found that driving cell differentiation caused a reduced local cell density in the basal layer, which in turn drove stem cell proliferation as contact inhibition was reduced. Whether this inhibition is through physical or 
chemical signalling, it is the differentiating progeny of the stem cells that feed back to suppress their proliferation. A recent study has suggested that the mechanism may be based on intra-tissue tension (Ning et al. 2021). Signals from stem cell progeny are also important in the activation of quiescent mammalian hair follicle stem cells. Transit-amplifying (TA) cells secrete the Hedgehog ligand Shh to activate the hair follicle stem cells (Hsu et al. 2014).

Given the importance of coupling cell loss to new cell production, both in normal homeostasis and in response to challenges, it is perhaps not surprising that progeny should feed back to the stem cells (Fig. 1a). This form of coupling would allow the status of the epithelium to be communicated continuously to the stem cells, enabling them in turn to tune the production of new cells according to demand. The importance of stem cell progeny in the niche shifts our understanding from that of a fixed, clearly defined anatomical niche to that of a niche in continuous flux, able to act as rheostat, dynamically adapting to challenges and maintaining balance (Hsu and Fuchs 2012).

\section{Being the Niche: Stem Cell-Derived Proteins as Niche Components}

In addition to producing differentiated progeny that comprises part of the stem cell niche, there are an increasing number of examples of stem cells expressing their own niche components. In some cases, these act as autocrine signals; in others, they may contribute to the local environment, shaping the extracellular matrix (ECM) and modulating paracrine signals. In the mammalian interfollicular epidermis, stem cell self-renewal is driven by autocrine Wnt signalling (Lim et al. 2013). Wnt4 and Wnt10a are expressed by stem cells in both mouse epidermis in vivo and cultured human epidermal stem cells (Radoja et al. 2006; Lim et al. 2013). In the epidermal appendages, autocrine FGF18 and BMP6 act as niche factors to maintain quiescence of hair follicle bulge stem cells (Kimura-Ueki et al. 2012).
One population of stem cells may in some contexts provide critical niche signals for another, for example, apoptotic hair follicle stem cells were recently shown to promote the proliferation of their non-apoptotic neighbours through Wnt signalling (Ankawa et al. 2021).

Similar mechanisms are found in the Drosophila intestine with Pvf2 acting as an autocrine stem cell maintenance factor. Overexpression of Pvf2 results in hyperproliferation, while depletion results in epithelial hypoplasia (Bond and Foley 2012). Increased expression of Pvf 2 in aged intestines has been associated with stem cell misregulation, and knockdown of Pvf2 blocks increased proliferation observed with age, illustrating the importance of stem cell niche contributions in maintaining homeostatic balance (Choi et al. 2008). Autocrine signalling is not limited to Pvf2; the cytokine JAK/STAT ligand Upd1 is required autonomously for stem/progenitor cell maintenance (Osman et al. 2012). As in mammalian epidermis, there is some evidence that autocrine $\mathrm{Wg}$ signalling may contribute to stem cell regulation in the Drosophila midgut, particularly in anterior regions that may be further from a source of paracrine Wingless signals (Fang et al. 2016).

The role of stem cells in secreting their own niche proteins is not limited to signalling pathway ligands but also includes the contribution of stem cells to ECM composition. ECM components have been described as possible stem cell markers in the epidermal hair follicle bulge (Watt and Fujiwara 2011), with a range of collagens showing increased expression in hair follicle stem cells (Morris et al. 2004) and epidermal label-retaining cells (Tumbar et al. 2004). Given that integrin expression and adhesive properties are well-established markers of epidermal stem cells and that Ilk signalling in epidermal progenitor cells has been linked to the composition of the ECM, this may in effect represent an autocrine signalling mechanism with stem cells secreting their own ECM substrate to promote their maintenance (Jones and Watt 1993; Jones et al. 1995; Morgner et al. 2015). This may be a conserved principle of epithelial stem cell niches as Drosophila midgut ISCs are also maintained by 
integrin signalling and express the ECM component laminin as an integrin ligand (Lin et al. 2013). In the mammalian intestine, both mesenchymal and epithelial cells contribute to the ECM components of the stem cell niche (Meran et al. 2017). Collagen VI is expressed in the basal epithelial layer of adult human intestine in the crypt region and in cultured human intestinal epithelial cells, suggesting it is produced either by the ISCs or their immediate progeny (Groulx et al. 2011). A similar pattern has been described for fibronectin in rat intestinal tissue and cultured cells (Quaroni et al. 1978), and some laminin subunits, such as $\alpha 2$, are expressed by epithelial cells and enriched around the crypt region (Lefebvre et al. 1999; Teller et al. 2007). Either by acting directly as autocrine signalling molecules or by creating a permissive physical environment, stem cellderived ECM components contribute significantly to epithelial niches.

Autocrine signalling from stem cells (Fig. 1b) may be broadly associated with positive feedback mechanisms, serving to keep stem cells in a primed state where the removal of inhibitory factors could lead to rapid proliferation, for example, in the context of repairing tissue damage. However, such mechanisms pose risks for unregulated proliferation, and as discussed below, there are many examples of autocrine signalling within tumours.

\section{$4 \quad$ Regulating the Niche: Stem Cell Signals to the Microenvironment}

In addition to autocrine signals, stem cells also express a range of paracrine signals that play important roles in the regulation of their environment. Stem cell progeny in many cases constitute a critical niche component regulating their parental stem cells; in turn, it is perhaps not surprising that stem cells also regulate their progeny. Drosophila ISCs express the Notch ligand Delta, which signals to differentiating EBs to promote enterocyte fate (Micchelli and Perrimon 2006; Ohlstein and Spradling 2006, 2007). Mammalian Delta-like 1 (DLL1) has been found to perform a similar function in mouse interfollicular epidermis, where it acts as a juxtacrine signal from stem cells in the basal layer to promote differentiation (Lowell et al. 2000). Recent work has identified an additional direct role for DLL1 in not only promoting differentiation of adjacent cells but actively inhibiting differentiation of the DLL1expressing stem cells. Cis-inhibition by DLL1 in the stem cells inhibits the Notch signalling pathway to block differentiation (Negri et al. 2019). In effect, DLL1 is acting as both an autocrine inhibitory signal to maintain stemness and a paracrine Notch-activating signal to promote progeny differentiation. This complexity of local signalling is also observed for the Wnt pathway: as discussed in the previous section, mouse interfollicular epidermal stem cells express Wnt ligands that act as autocrine signals to promote stem cell fate; but they also secrete paracrine Wnt inhibitors that promote differentiation of their progeny (Lim et al. 2013). In addition to regulating cellular niche components, stem cells may also impact ECM composition through expression of regulators such as Timp2 in epidermal stem cells (Morris et al. 2004).

Expression profiling has identified a number of secreted proteins expressed by Drosophila ISCs that may act to regulate the local microenvironment (Doupé et al. 2018). The insulin-like peptide Ilp6 is expressed in the ISCs and was found to repress stem cell turnover, suggesting a non-autocrine role since ISC insulin signalling has previously been shown to be necessary for homeostatic turnover and growth (Choi et al. 2011; O'Brien et al. 2011). This study also identified the TNF ligand Eiger as a stem cellexpressed protein that promotes stem cell proliferation, possibly through indirect effects on stem cell enterocyte progeny (Doupé et al. 2018). Another group has further demonstrated that Eiger may be involved in both autocrine and paracrine positive feedback to promote stem cell divisions (Tamamouna et al. 2020).

Stem cell signalling to the microenvironment also plays a significant role in homeostatic turnover of the lung and airway epithelia. As in the examples above, Notch signalling from stem cells to their progeny occurs in the lung epithelium, but 
it is required for the maintenance of secretory progeny rather than to promote differentiation (Pardo-Saganta et al. 2015). In co-culture assays, human airway stem/progenitor cells express VEGF-A and FGF ligands, which signal locally to endothelial cells to promote their function as sources of niche factors, such as MMP14 for the airway stem cell growth (Curradi et al. 2012; Ding et al. 2015).

Given the dynamic nature of epithelial turnover in homeostasis and the need to closely coordinate cell loss, differentiation and proliferation, it is perhaps not surprising that stem cells communicate their status to the niche, including their own progeny (Fig. 1c). In effect, this allows stem cells to answer back to the microenvironment, allowing a precise two-way feedback with the niche to offer a range of mechanisms that support balanced turnover.

\section{Subverting the Niche: Carcinogenesis and Metastasis}

The ability of stem cells to form, regulate or act as niche cells can be a double-edged sword. The potency that enables precise coordinated regulation in homeostasis and repair can be subverted in diseases such as cancers. Mutations not only trigger autonomous effects in the tumour itself but also impact the ability of the cancer stem cells (CSCs) or tumour-initiating cells (TICs) to communicate to and regulate their surroundings. A great deal of research has focused on understanding how tumour cells remodel their local microenvironment to promote their growth, invasion and metastasis at the expense of normal stem cells (Prager et al. 2019; López de Andrés et al. 2020; Winkler et al. 2020; Oshimori et al. 2021). CSCs have been shown to produce niche factors that either directly promote their self-renewal and survival or help create a favourable environment for cancer progression and invasion (Plaks et al. 2015). This complex network of cross-talk and signalling involves many of the key components of the tumour microenvironment, including mesenchymal stem cells, cancer-associated fibroblasts, immune cells and endothelial cells, along with the extracellular matrix and a wide range of cytokines.

The importance of cancer cell progeny as components of the niche that drive proliferation has been explored in the Drosophila midgut epithelium. In a Drosophila intestinal tumour model driven by loss of Notch function, which blocks differentiation, both autocrine and paracrine signals are responsible for tumour growth (Patel et al. 2015). Autocrine EGF signalling supports initial growth, but as tumour expansion displaces differentiated epithelial cells, these cancer cell progeny express cytokine ligands to further drive tumour growth. An alternative tumour model, in which loss of the transcription factor Sox 21 a causes accumulation of undifferentiated stem cell progeny, shows similar principles, with stem cell progeny expressing cytokine ligands, EGF ligands and Pvf2 to drive proliferation and matrix metalloproteases to drive invasion (Zhai et al. 2015; Chen et al. 2016). There is an extensive literature on tumour heterogeneity and the significance of communication between subclones within a tumour (Tabassum and Polyak 2015). In some cases, this is likely to include communication between cancer stem cells and their own progeny, for example, in a p53-null mouse model of breast cancer, mesenchymallike progeny signal to their tumour-initiating cell parents to promote CSC self-renewal (Brooks and Wicha 2015; Zhang et al. 2015).

Autocrine signalling also plays key roles in the maintenance and proliferation of cancer stem cells. In SOS-driven or DMBA/TPA-induced mouse skin tumours, autocrine VEGF signalling drives tumour cell proliferation, and loss of VEGF or its receptor in the epithelium is sufficient to block tumour growth (Lichtenberger et al. 2010; Beck et al. 2011). Autocrine VEGF has roles in cell survival, proliferation or migration in a range of tumours, including the lung (Chatterjee et al. 2013; Barr et al. 2015), breast (Perrot-Applanat and Di Benedetto 2012) and oesophagus (Zhang et al. 2014). Oesophageal cancer stem cells upregulate both the cytokine CXCL2 and its receptor CXCR4 to promote invasion and metastasis (Wang et al. 2017). Advanced skin squamous cell carcinomas 
express autocrine SDF-1 that signals through CXCR4 and CXCR7 to promote metastasis (Bernat-Peguera et al. 2019). Ovarian carcinomas express autocrine Interleukin-6 (IL-6), and inhibition of its receptor reduces metastasis (Mehner et al. 2020). Autocrine FGF signalling occurs in squamous cell carcinomas of the lung and head and neck (Gao et al. 2019; Li et al. 2020) and enables adaptive resistance to BRAF/MEK inhibition in BRAF tumours (Wang et al. 2019). Autocrine TGF $\beta$ signalling has been implicated in breast, colorectal and ovarian cancers (Matsumoto et al. 2018; Nakano et al. 2019; Woosley et al. 2019). IL-6 is also an important autocrine factor, in addition to its paracrine roles in regulating the stem cell niche (Yeh et al. 2006; Sansone et al. 2007; Sasser et al. 2007). The ECM protein periostin has been identified as another stem cell-secreted protein that promotes cancer stem cell fate in breast, colon and ovarian cancer through autocrine integrin signalling (Gillan et al. 2002; Bao et al. 2004; Lambert et al. 2016). Periostin is also expressed in mouse hair follicle stem cells, suggesting a role in normal stem cells that is subverted in cancer (Morris et al. 2004).

In addition to acting as a positive niche for their own maintenance through autocrine signalling, cancer stem cells can act as a restrictive niche to limit the proliferation and maintenance of normal stem cells. Three recent studies found that intestinal tumours alter the niche not only to promote their own growth but to drive the loss of adjacent wild-type stem cells. Mammalian ISCs mutant for Apc secrete Wnt antagonists such as Notum to inhibit Wnt signalling in wild-type ISCs, suppressing their proliferation and promoting their differentiation (Flanagan et al. 2021; van Neerven et al. 2021). The mutant ISCs are therefore acting as a repressive niche for normal ISC growth, but as the Apc mutant cells are no longer dependent on extrinsic Wnt signals, their own growth is not impeded. This remodelling was not specific to Apc cells or Wnt signalling, as clones of intestinal cells expressing KRAS/PI3K oncogenes have also been shown to suppress the growth of adjacent wild-type clones by secretion of BMP ligands (Yum et al. 2021). The Simons group also showed that in addition to directly acting as a repressive niche, cancer stem cells can regulate other niche components, indirectly repressing Wnt signalling in wild-type stem cells by remodelling of the stroma. Collectively, these three papers demonstrate that cancer stem cells remodel the local microenvironment to enhance their own competitive advantage over wild-type stem cells.

CSC- or TIC-derived signals play critical roles in regulating their own niche through recruitment or induction of cells in the microenvironment to express niche signals that promote tumour properties. CSCs in breast cancer and skin tumours secrete VEGF-A, which acts on endothelial cells to promote angiogenesis and the formation of a perivascular niche (Beck et al. 2011; Jiang et al. 2020). In addition to its role in promoting tumour endothelial niche function, mouse colon carcinoma-derived VEGF-A has also been shown to regulate T-cell immune checkpoints, creating an immune-protective environment (Voron et al. 2015). Squamous cell carcinoma TICs express the cytokine IL-33, which induces differentiation of macrophages that express TGF $\beta$ to promote cancer progression (Taniguchi et al. 2020). A similar loop is observed between breast CSCs and mesenchymal stem cells involving reciprocal IL-6 and CXCL7 signalling (Liu et al. 2011). Tumour-derived signals can also induce cancer-associated fibroblasts (CAFs), including inflammatory CAFs via IL- $1 \alpha$ and myofibroblastic CAFs via TGF $\beta$ (Biffi et al. 2019). In the Drosophila midgut, Apc mutant cells subvert the normal EGF-driven feedback circuit that acts to couple cell loss to ISC proliferation by inducing rhomboid in adjacent cells to drive constitutive secretion of active EGF ligands (Ngo et al. 2020). Remodelling of the ECM elements of the niche is also critical in tumour development and progression, with contributions from both tumour cells themselves expressing ECM components or regulators such as MMPs and from tumour-associated cells, particularly CAFs (Winkler et al. 2020). In addition to its autocrine roles, the ECM component periostin is expressed by CAFs in the stroma of ovarian cancer in response to TGF $\beta 1$ signalling from the 
tumour cells and promotes metastasis through integrin signalling (Yue et al. 2021).

In summary, cancer stem cells extensively remodel their niche in order to facilitate tumour progression and cancer cell survival through complex networks of interactions involving all of the contributions seen in the homeostatic niche (Fig. 1d). As a result, the cancer secretome is increasingly taking on therapeutic significance (López de Andrés et al. 2020). The multipotency and tight homeostatic regulation that characterizes stem cells and maintains tissue integrity is overturned in the CSC niche, promoting adverse effects of an otherwise beneficial characteristic of normal tissue stem cells.

\section{Conclusions}

The importance of stem cell-derived secreted proteins and stem cell progeny in the niche is increasingly recognized in the context of cancer cells. However, the extent to which this represents the misregulation of signalling circuits used in normal stem cell regulation is unclear. Studies from a range of systems have shown the significance of these mechanisms in normal homeostasis and tissue repair. Further work is needed to explore the extent of stem cell signalling as critical to the niche and the logic of two-way interactions between stem cells and the surrounding microenvironment. Coupling both at the level of two-way signalling between stem cells and the niche and between cell production and loss through niche roles of stem cell progeny provides mechanisms to maintain balance. Given the highly dynamic nature of these links, the development and application of novel approaches to study homeostasis in real time will be critical to explore the mechanisms of homeostasis and how it is perturbed. Many of the known mechanisms appear to be conserved from the relatively simple invertebrate model of the Drosophila midgut to mammalian systems, suggesting that complementary studies in both basic model organisms and more complex mammalian systems will be key to elucidating the full logic of these complex interactions.
Acknowledgements We thank Pedro Saavedra for his comments on the manuscript. This work was supported by a Newcastle Liverpool Durham BBSRC-DTP [BB/M011186/1] PhD studentship and the Academy of Medical Sciences/Wellcome Trust/Government Department of Business, Energy and Industrial Strategy/ British Heart Foundation/Diabetes UK Springboard Award [SBF005\1022].

\section{References}

Amcheslavsky A et al (2014) Enteroendocrine cells support intestinal stem-cell-mediated homeostasis in Drosophila. Cell Rep 9(1):32-39. https://doi.org/10.1016/ j.celrep.2014.08.052

Ankawa R et al (2021) Apoptotic cells represent a dynamic stem cell niche governing proliferation and tissue regeneration. Dev Cell 56(13):1900-1916.e5. https:// doi.org/10.1016/j.devcel.2021.06.008

Aoki R et al (2016) Foxl1-expressing mesenchymal cells constitute the intestinal stem cell niche. Cell Mol Gastroenterol Hepatol 2(2):175-188. https://doi.org/ 10.1016/j.jcmgh.2015.12.004

Bao S et al (2004) Periostin potently promotes metastatic growth of colon cancer by augmenting cell survival via the Akt/PKB pathway. Cancer Cell 5(4):329-339. https://doi.org/10.1016/S1535-6108(04)00081-9

Barker $\mathrm{N}$ et al (2007) Identification of stem cells in small intestine and colon by marker gene Lgr5. Nature 449(7165):1003-1007. https://doi.org/10.1038/ nature06196

Barr MP et al (2015) Vascular endothelial growth factor is an autocrine growth factor, signaling through neuropilin-1 in non-small cell lung cancer. Mol Cancer 14(1):45. https://doi.org/10.1186/s12943-015-0310-8

Beck B et al (2011) A vascular niche and a VEGF-Nrp1 loop regulate the initiation and stemness of skin tumours. Nature 478(7369):399-403. https://doi.org/ 10.1038 /nature 10525

Bernat-Peguera A et al (2019) PDGFR-induced autocrine SDF-1 signaling in cancer cells promotes metastasis in advanced skin carcinoma. Oncogene 38(25):5021-5037. https://doi.org/10.1038/s41388019-0773-y

Biffi $\mathrm{G}$ et al (2019) IL1-induced JAK/STAT signaling is antagonized by TGF $\beta$ to shape CAF heterogeneity in pancreatic ductal adenocarcinoma. Cancer Discov 9(2):282-301. https://doi.org/10.1158/2159-8290.CD18-0710

Biteau B, Jasper H (2011) EGF signaling regulates the proliferation of intestinal stem cells in Drosophila. Development 138(6):1045-1055. https://doi.org/10. 1242/dev.056671

Biteau B, Jasper H (2014) Slit/Robo signaling regulates cell fate decisions in the intestinal stem cell lineage of Drosophila. Cell Rep 7(6):1867-1875. https://doi.org/ 10.1016/j.celrep.2014.05.024 
Bond D, Foley E (2012) Autocrine platelet-derived growth factor-vascular endothelial growth factor receptorrelated (Pvr) pathway activity controls intestinal stem cell proliferation in the adult Drosophila midgut. J Biol Chem 287(33):27359-27370. https://doi.org/10.1074/ jbc.M112.378018

Brooks MD, Wicha MS (2015) Tumor Twitter: cellular communication in the breast cancer stem cell niche. Cancer Discov 5(5):469-471. https://doi.org/10.1158/ 2159-8290.CD-15-0327

Chacón-Martínez CA, Koester J, Wickström SA (2018) Signaling in the stem cell niche: regulating cell fate, function and plasticity. Development 145(15). https:// doi.org/10.1242/dev.165399

Chatterjee S et al (2013) Tumor VEGF:VEGFR2 autocrine feed-forward loop triggers angiogenesis in lung cancer. J Clin Invest 123(4):1732-1740. https://doi.org/10. 1172/JCI65385

Chen J et al. (2016) A feedback amplification loop between stem cells and their progeny promotes tissue regeneration and tumorigenesis. eLife. Edited by AC Spradling. eLife Sciences Publications, Ltd, 5:e14330. https://doi.org/10.7554/eLife.14330

Choi N-H et al (2008) Age-related changes in Drosophila midgut are associated with PVF2, a PDGF/VEGF-like growth factor. Aging Cell 7(3):318-334. https://doi. org/10.1111/j.1474-9726.2008.00380.x

Choi NH, Lucchetta E, Ohlstein B (2011) Nonautonomous regulation of Drosophila midgut stem cell proliferation by the insulin-signaling pathway. PNAS 108(46):18702-18707. https://doi.org/10.1073/pnas. 1109348108/-/DCSupplemental.www.pnas.org/cgi/ doi/10.1073/pnas.1109348108

Cordero JB et al (2012) Inducible progenitor-derived Wingless regulates adult midgut regeneration in Drosophila. EMBO J 31(19):3901-3917. https://doi.org/ 10.1038/emboj.2012.248

Curradi G et al (2012) Airway basal cell vascular endothelial growth factor-mediated cross-talk regulates endothelial cell-dependent growth support of human airway basal cells. Cell Mol Life Sci 69(13):2217-2231. https://doi.org/10.1007/s00018-012-0922-8

Degirmenci B et al (2018) GLI1-expressing mesenchymal cells form the essential Wnt-secreting niche for colon stem cells. Nature 558(7710):449-453. https://doi.org/ 10.1038/s41586-018-0190-3

Ding B-S et al (2015) Endothelial MMP14 is required for endothelial-dependent growth support of human airway basal cells. J Cell Sci 128(16):2983-2988. https://doi.org/10.1242/jcs. 168179

Doupé DP et al (2018) Drosophila intestinal stem and progenitor cells are major sources and regulators of homeostatic niche signals. Proc Natl Acad Sci 115(48):12218-12223. https://doi.org/10.1073/pnas. 1719169115

Durand A et al (2012) Functional intestinal stem cells after Paneth cell ablation induced by the loss of transcription factor Math1 (Atoh1). Proc Natl Acad Sci
109(23):8965-8970. https://doi.org/10.1073/pnas. 1201652109

Fang HY, Martinez-Arias A, de Navascués J (2016) Autocrine and paracrine wingless signalling in the drosophila midgut by both continuous gradient and asynchronous bursts of wingless expression [version 1; referees: 3 approved with reservations]. F1000Research 5(May):1-21. https://doi.org/10. 12688/F1000RESEARCH.8170.1

Flanagan DJ et al (2021) NOTUM from Apc-mutant cells biases clonal competition to initiate cancer. Nature 594(7863):430-435. https://doi.org/10.1038/s41586$021-03525-\mathrm{z}$

Gao L et al (2019) FGF19 amplification reveals an oncogenic dependency upon autocrine FGF19/FGFR4 signaling in head and neck squamous cell carcinoma. Oncogene 38(13):2394-2404. https://doi.org/10.1038/ s41388-018-0591-7

Gillan L et al (2002) Periostin secreted by epithelial ovarian carcinoma is a ligand for $\alpha_{V} \beta_{3}$ and $\alpha_{V} \beta_{5}$ Integrins and promotes cell motility. Cancer Res 62(18):5358-5364. Available at: http://cancerres. aacrjournals.org/content/62/18/5358.abstract

Groulx J-F et al (2011) Collagen VI is a basement membrane component that regulates epithelial cell-fibronectin interactions. Matrix Biol 30(3):195-206. https://doi.org/10.1016/j.matbio.2011.03.002

Hanahan D, Weinberg RA (2017) Hallmarks of cancer: the next generation. Cell 144(5):646-674. https://doi.org/ 10.1016/j.cell.2011.02.013

Hsu Y-C, Fuchs E (2012) A family business: stem cell progeny join the niche to regulate homeostasis. Nat Rev Mol Cell Biol 13(2):103-114. https://doi.org/10. 1038/nrm3272

Hsu YC, Li L, Fuchs E (2014) Transit-amplifying cells orchestrate stem cell activity and tissue regeneration. Cell 157(4):935-949. https://doi.org/10.1016/j.cell. 2014.02.057

Jiang H et al (2009) Cytokine/Jak/Stat signaling mediates regeneration and homeostasis in the Drosophila midgut. Cell 137(7):1343-1355. https://doi.org/10.1016/j. cell.2009.05.014

Jiang $\mathrm{H}$ et al (2011) EGFR/Ras/MAPK signaling mediates adult midgut epithelial homeostasis and regeneration in Drosophila. Cell Stem Cell 8(1):84-95. https://doi.org/ 10.1016/j.stem.2010.11.026

Jiang $\mathrm{H}$ et al (2020) Jagged1-Notch1-deployed tumor perivascular niche promotes breast cancer stem cell phenotype through Zeb1. Nat Commun 11(1):5129. https://doi.org/10.1038/s41467-020-18860-4

Jones PH, Watt FM (1993) Separation of human epidermal stem cells from transit amplifying cells on the basis of differences in integrin function and expression. Cell 73(4):713-724. https://doi.org/10.1016/0092-8674 (93)90251-K

Jones PH, Harper S, Watt FM (1995) Stem cell patterning and fate in human epidermis. Cell 80(1):83-93. https:// doi.org/10.1016/0092-8674(95)90453-0 
Karpowicz P, Perez J, Perrimon N (2010) The hippo tumor suppressor pathway regulates intestinal stem cell regeneration. Development 137(24):4135-4145. https://doi.org/10.1242/dev.060483

Kim T-H, Escudero S, Shivdasani RA (2012) Intact function of Lgr5 receptor-expressing intestinal stem cells in the absence of Paneth cells. Proc Natl Acad Sci:201113890. https://doi.org/10.1073/pnas. 1113890109

Kimura-Ueki M et al (2012) Hair cycle resting phase is regulated by cyclic epithelial FGF18 signaling. J Investig Dermatol 132(5):1338-1345. https://doi.org/ 10.1038/jid.2011.490

Lambert AW et al (2016) Tumor cell-derived periostin regulates cytokines that maintain breast cancer stem cells. Mol Cancer Res 14(1):103-113. https://doi.org/ 10.1158/1541-7786.MCR-15-0079

Lefebvre O et al (1999) Developmental expression and cellular origin of the laminin $\alpha 2, \alpha 4$, and $\alpha 5$ chains in the intestine. Dev Biol 210(1):135-150. https://doi. org/10.1006/dbio.1999.9270

Li F et al (2020) Enhanced autocrine FGF19/FGFR4 signaling drives the progression of lung squamous cell carcinoma, which responds to mTOR inhibitor AZD2104. Oncogene 39(17):3507-3521. https://doi. org/10.1038/s41388-020-1227-2

Liang J et al (2017) Feedback regulation of steady-state epithelial turnover and organ size. Nature 548(7669):588-591. https://doi.org/10.1038/ nature 23678

Lichtenberger BM et al (2010) Autocrine VEGF signaling synergizes with EGFR in tumor cells to promote epithelial cancer development. Cell 140(2):268-279. https://doi.org/10.1016/j.cell.2009.12.046

Lim X et al (2013) Interfollicular epidermal stem cells selfrenew via autocrine Wnt signaling. Science (New York, NY) 342(6163):1226-1230. https://doi. org/10.1126/science. 1239730

Lin $\mathrm{G}$ et al (2013) Integrin signaling is required for maintenance and proliferation of intestinal stem cells in Drosophila. Dev Biol 377(1):177-187. https://doi.org/ 10.1016/j.ydbio.2013.01.032

Liu S et al (2011) Breast cancer stem cells are regulated by mesenchymal stem cells through cytokine networks. Cancer Res 71(2):614-624. https://doi.org/10.1158/ 0008-5472.CAN-10-0538

López de Andrés J et al (2020) Cancer stem cell secretome in the tumor microenvironment: a key point for an effective personalized cancer treatment. J Hematol Oncol 13(1):136. https://doi.org/10.1186/s13045-02000966-3

Lowell S et al (2000) Stimulation of human epidermal differentiation by Delta-Notch signalling at the boundaries of stem-cell clusters. Curr Biol 10(9):491-500. https://doi.org/10.1016/S0960-9822 (00)00451-6

Matsumoto T et al (2018) TGF- $\beta$-mediated LEFTY/Akt/ GSK-3 $\beta$ /snail axis modulates epithelial-mesenchymal transition and cancer stem cell properties in ovarian clear cell carcinomas. Mol Carcinog 57(8):957-967. https://doi.org/10.1002/mc.22816

Mehner C et al (2020) Targeting an autocrine IL-6SPINK1 signaling axis to suppress metastatic spread in ovarian clear cell carcinoma. Oncogene 39(42):6606-6618. https://doi.org/10.1038/s41388020-01451-4

Meran L, Baulies A, Li VSW (2017) Intestinal stem cell niche: the extracellular matrix and cellular components. Stem Cells International. Edited by K Liu Hindawi 2017:7970385. https://doi.org/10.1155/ 2017/7970385

Mesa KR et al (2018) Homeostatic epidermal stem cell self-renewal is driven by local differentiation. Cell Stem Cell 23(5):677-686.e4. https://doi.org/10.1016/ j.stem.2018.09.005

Micchelli CA, Perrimon N (2006) Evidence that stem cells reside in the adult Drosophila midgut epithelium. Nature 439(7075):475-479. https://doi.org/10.1038/ nature04371

Morgner J et al (2015) Integrin-linked kinase regulates the niche of quiescent epidermal stem cells. Nat Commun 6(1):8198. https://doi.org/10.1038/ncomms9198

Morris RJ et al (2004) Capturing and profiling adult hair follicle stem cells. Nat Biotechnol 22(4):411-417. https://doi.org/10.1038/nbt950

Nakano M et al (2019) Dedifferentiation process driven by TGF-beta signaling enhances stem cell properties in human colorectal cancer. Oncogene 38(6):780-793. https://doi.org/10.1038/s41388-018-0480-0

Negri VA et al (2019) Delta-like 1-mediated cis-inhibition of Jagged $1 / 2$ signalling inhibits differentiation of human epidermal cells in culture. Sci Rep 9(1):10825. https:// doi.org/10.1038/s41598-019-47232-2

Ngo S et al (2020) Disruption of EGF feedback by intestinal tumors and neighboring cells in Drosophila. Curr Biol 30(8):1537-1546.e3. https://doi.org/10.1016/j. cub.2020.01.082

Ning W et al (2021) Differentiated daughter cells regulate stem cell proliferation and fate through intra-tissue tension. Cell Stem Cell 28(3):436-452.e5. https://doi. org/10.1016/j.stem.2020.11.002

O'Brien LE et al (2011) Altered modes of stem cell division drive adaptive intestinal growth. Cell 147(3):603-614. https://doi.org/10.1016/j.cell.2011. 08.048

Ohlstein B, Spradling A (2006) The adult Drosophila posterior midgut is maintained by pluripotent stem cells. Nature 439(7075):470-474. https://doi.org/10. 1038/nature04333

Ohlstein B, Spradling A (2007) Multipotent Drosophila intestinal stem cells specify daughter cell fates by differential notch signaling. Science (New York, NY) 315(5814):988-992. https://doi.org/10.1126/science. 1136606

Oshimori N, Guo Y, Taniguchi S (2021) An emerging role for cellular crosstalk in the cancer stem cell niche. $\mathrm{J}$ Pathol 254(4):384-394. https://doi.org/10.1002/path. 5655 
Osman D et al (2012) Autocrine and paracrine unpaired signaling regulate intestinal stem cell maintenance and division. J Cell Sci 125(Pt 24):5944-5949. https://doi. org/10.1242/jcs. 113100

Pardo-Saganta A et al (2015) Parent stem cells can serve as niches for their daughter cells. Nature 523(7562): 597-601. https://doi.org/10.1038/nature14553

Patel PH, Dutta D, Edgar BA (2015) Niche appropriation by Drosophila intestinal stem cell tumours. Nat Cell Biol 17(9):1182-1192. https://doi.org/10.1038/ ncb3214

Pentinmikko N et al (2019) Notum produced by Paneth cells attenuates regeneration of aged intestinal epithelium. Nature 571(7765):398-402. https://doi.org/10. 1038/s41586-019-1383-0

Perrot-Applanat M, Di Benedetto M (2012) Autocrine functions of VEGF in breast tumor cells. Cell Adhes Migr 6(6):547-553. https://doi.org/10.4161/cam. 23332

Plaks V, Kong N, Werb Z (2015) The cancer stem cell niche: how essential is the niche in regulating stemness of tumor cells? Cell Stem Cell 16(3):225-238. https:// doi.org/10.1016/j.stem.2015.02.015

Prager BC et al (2019) Cancer stem cells: the architects of the tumor ecosystem. Cell Stem Cell 24(1):41-53. https://doi.org/10.1016/j.stem.2018.12.009

Quaroni A, Isselbacher KJ, Ruoslahti E (1978) Fibronectin synthesis by epithelial crypt cells of rat small intestine. Proc Natl Acad Sci 75(11):5548-5552. https://doi.org/ 10.1073/pnas.75.11.5548

Radoja N et al (2006) Transcriptional profiling of epidermal differentiation. Physiol Genomics 27(1):65-78. https://doi.org/10.1152/physiolgenomics.00031.2006

Sansone P et al (2007) IL-6 triggers malignant features in mammospheres from human ductal breast carcinoma and normal mammary gland. $J$ Clin Invest 117(12):3988-4002. https://doi.org/10.1172/JCI32533

Santos AJM et al (2018) The intestinal stem cell niche: homeostasis and adaptations. Trends Cell Biol 28(12):1062-1078. https://doi.org/10.1016/j.tcb.2018. 08.001

Sasser AK et al (2007) Interleukin-6 is a potent growth factor for ER- $\alpha$-positive human breast cancer. FASEB J 21(13):3763-3770. https://doi.org/10.1096/fj.07$8832 \mathrm{com}$

Sato T et al (2009) Single Lgr5 stem cells build cryptvillus structures in vitro without a mesenchymal niche. Nature 459(7244):262-265. https://doi.org/10.1038/ nature 07935

Sato T et al (2011) Paneth cells constitute the niche for Lgr5 stem cells in intestinal crypts. Nature 469(7330):415-418. https://doi.org/10.1038/ nature09637

Scadden DT (2014) Nice Neighborhood: emerging concepts of the stem cell niche. Cell 157(1):41-50. https://doi.org/10.1016/j.cell.2014.02.013

Shoshkes-Carmel M et al (2018) Subepithelial telocytes are an important source of Wnts that supports intestinal crypts. Nature 557(7704):242-246. https://doi.org/10. 1038/s41586-018-0084-4

Tabassum DP, Polyak K (2015) Tumorigenesis: it takes a village. Nat Rev Cancer 15(8):473-483. https://doi. org/10.1038/nrc3971

Tamamouna V et al (2020) Evidence of two types of balance between stem cell mitosis and enterocyte nucleus growth in the Drosophila midgut. Development 147(11). https://doi.org/10.1242/dev.189472

Taniguchi S et al (2020) Tumor-initiating cells establish an IL-33-TGF- $\beta$ niche signaling loop to promote cancer progression. Science 369(6501):eaay 1813. https://doi. org/10.1126/science.aay 1813

Teller IC et al (2007) Laminins in the developing and adult human small intestine: relation with the functional absorptive unit. Dev Dyn 236(7):1980-1990. https:// doi.org/10.1002/dvdy.21186

Tian A, Jiang J (2014) Intestinal epithelium-derived BMP controls stem cell self-renewal in Drosophila adult midgut. eLife. Edited by U Banerjee 3:e01857. https://doi.org/10.7554/eLife.01857

Tumbar $\mathrm{T}$ et al (2004) Defining the epithelial stem cell niche in skin. Science 303(5656):359-363. https://doi. org/10.1126/science.1092436

Valenta $\mathrm{T}$ et al (2016) Wnt ligands secreted by subepithelial mesenchymal cells are essential for the survival of intestinal stem cells and gut homeostasis. Cell Rep 15(5):911-918. https://doi.org/10.1016/j. celrep.2016.03.088

van Neerven SM et al (2021) Apc-mutant cells act as supercompetitors in intestinal tumour initiation. Nature 594(7863):436-441. https://doi.org/10.1038/s41586021-03558-4

Voron $\mathrm{T}$ et al (2015) VEGF-A modulates expression of inhibitory checkpoints on CD8+ T cells in tumors. $\mathrm{J}$ Exp Med 212(2):139-148. https://doi.org/10.1084/ jem.20140559

Wang X et al (2017) Stem cell autocrine CXCL12/CXCR4 stimulates invasion and metastasis of esophageal cancer. Oncotarget 8(22) Available at: https://www. oncotarget.com/article/15254/text/

Wang VE et al (2019) Adaptive resistance to dual BRAF/ MEK inhibition in BRAF-driven tumors through autocrine FGFR pathway activation. Clin Cancer Res 25(23):7202-7217. https://doi.org/10.1158/ 1078-0432.CCR-18-2779

Watt FM, Fujiwara H (2011) Cell-extracellular matrix interactions in normal and diseased skin. Cold Spring Harb Perspect Biol 3(4):1-14. https://doi.org/10.1101/ cshperspect.a005124

Winkler J et al (2020) Concepts of extracellular matrix remodelling in tumour progression and metastasis. Nat Commun 11(1):5120. https://doi.org/10.1038/s41467020-18794-x

Woosley AN et al (2019) TGF $\beta$ promotes breast cancer stem cell self-renewal through an ILEI/LIFR signaling axis. Oncogene 38(20):3794-3811. https://doi.org/10. 1038/s41388-019-0703-z 
$\mathrm{Xu} \mathrm{N}$ et al (2011) EGFR, Wingless and JAK/STAT signaling cooperatively maintain drosophila intestinal stem cells. Dev Biol 354(1):31-43. https://doi.org/10. 1016/j.ydbio.2011.03.018

Yeh H-H et al (2006) Autocrine IL-6-induced Stat3 activation contributes to the pathogenesis of lung adenocarcinoma and malignant pleural effusion. Oncogene 25(31):4300-4309. https://doi.org/10.1038/sj.onc. 1209464

Yue $\mathrm{H}$ et al (2021) Stromal POSTN induced by TGF- $\beta 1$ facilitates the migration and invasion of ovarian cancer. Gynecol Oncol 160(2):530-538. https://doi.org/10. 1016/j.ygyno.2020.11.026

Yum MK et al (2021) Tracing oncogene-driven remodelling of the intestinal stem cell niche. Nature 594(7863):442-447. https://doi.org/10.1038/s41586021-03605-0
Zhai Z et al (2015) Accumulation of differentiating intestinal stem cell progenies drives tumorigenesis. Nat Commun 6:10219. https://doi.org/10.1038/ncomms10219

Zhang Q et al (2014) Autocrine VEGF Signaling promotes proliferation of neoplastic Barrett's epithelial cells through a PLC-dependent pathway. Gastroenterology 146(2):461-472.e6. https://doi.org/10.1053/j.gastro. 2013.10.011

Zhang $\mathrm{M}$ et al (2015) Intratumoral heterogeneity in a Trp53-null mouse model of human breast cancer. Cancer Discov 5(5):520-533. https://doi.org/10.1158/ 2159-8290.CD-14-1101

Zhou F et al (2013) The UPD3 cytokine couples environmental challenge and intestinal stem cell division through modulation of JAK/STAT signaling in the stem cell microenvironment. Dev Biol 373(2):383-393. https:// doi.org/10.1016/j.ydbio.2012.10.023

Open Access This chapter is licensed under the terms of the Creative Commons Attribution 4.0 International License (http://creativecommons.org/licenses/by/4.0/), which permits use, sharing, adaptation, distribution and reproduction in any medium or format, as long as you give appropriate credit to the original author(s) and the source, provide a link to the Creative Commons license and indicate if changes were made.

The images or other third party material in this chapter are included in the chapter's Creative Commons license, unless indicated otherwise in a credit line to the material. If material is not included in the chapter's Creative Commons license and your intended use is not permitted by statutory regulation or exceeds the permitted use, you will need to obtain permission directly from the copyright holder. 